\title{
Fraturas complexas do sacro com dissociação espinopélvica tratadas cirurgicamente com fixação iliolombar*
}

\section{Complex Fractures of the Sacrum with Spinopelvic Dissociation Treated Surgically with Iliolumbar Fixation}

\author{
Alberto Kazuo Miyamoto $^{10}$ Xavier Soler I Graells ${ }^{1,2}$ André Luis Sebben ${ }^{1}$ Marcel Luiz Benato ${ }^{1}$ \\ Pedro Grein Del Santoro ${ }^{1}$ Álynson Larocca Kulcheski ${ }^{1}$ \\ ${ }^{1}$ Grupo de Cirurgia da Coluna, Hospital do Trabalhador, Curitiba, PR, Brasil \\ 2 Departamento de Ortopedia e Traumatologia, Hospital das Clínicas, \\ Universidade Federal do Paraná (UFPR), Curitiba, PR, Brasil \\ Endereço para correspondência Alberto Kazuo Miyamoto, Hospital \\ do Trabalhador, Cirurgia da Coluna, Rua Martin Afonso 1.900, apto. \\ 404, Bigorrilho, Curitiba, PR, 80730-030, Brasil \\ (e-mail: betokm@hotmail.com).
}

Rev Bras Ortop 2020;55(3):304-309.

\section{Resumo}

\section{Palavras-chave}

- sacro

- ferimentos e lesões

- traumatismos da coluna vertebral

- fraturas da coluna vertebral

\section{Abstract}

Objetivo Analisar uma série de casos de fraturas complexas do sacro com dissociação espinopélvica tratados cirurgicamente com fixação iliolombar, e revisar a literatura médica existente.

Métodos Para a análise, foram avaliados retrospectivamente os prontuários médicos de casos operados utilizando a técnica de Schildhauer et al para fixação. O período de acompanhamento foi de pelo menos 12 meses. Os resultados funcionais foram avaliados por meio da escala visual analógica (EVA) de dor e do índice de incapacidade de Oswestry, versão 2.0. Os dados foram comparados com os da literatura médica existente.

Resultados Seis casos foram analisados, sendo que quatro evoluíram com incapacidade moderada, um, com incapacidade mínima, e um, com incapacidade grave. Três casos que apresentavam déficit neurológico obtiveram melhora significativa. Apenas um caso evoluiu com complicação tromboembólica pulmonar.

Conclusão A técnica de Schildhauer et al é eficiente para a fixação de fraturas complexas de sacro com dissociação espinopélvica. Os pacientes evoluíram com bons resultados funcionais. A liberação precoce de sustentação de carga demonstrou-se segura com o uso deste tratamento.

Objective To analyze a series of cases of complex fractures of the sacrum with spinopelvic dissociation surgically treated with iliolumbar fixation, and to review the existing medical literature.

Methods For the analysis, the medical records of the cases operated using the Schildhauer et al technique for fixation were retrospectively evaluated, and followed up

Trabalho desenvolvido no Grupo de Cirurgia da Coluna, Hospital do Trabalhador, Curitiba, PR, Brasil.

recebido

23 de Outubro de 2018

aceito

12 de Março de 2019
DOI https://doi.org/

$10.1055 / \mathrm{s}-0039-3402474$. ISSN 0102-3616.
Copyright $\odot 2020$ by Sociedade Brasileira License terms de Ortopedia e Traumatologia. Published by Thieme Revinter Publicações Ltda, Rio de Janeiro, Brazil 


\section{Keywords}

- sacrum

- wounds and injuries

- spinal injuries

- spinal fractures for at least 12 months. The functional results were assessed using the visual analog scale (VAS) for pain and the Oswestry disability index, version 2.0. The data were compared with those of the existing medical literature.

Results Six cases were analyzed, four of which evolved with moderate disability, one, with minimal disability, and one, with severe disability. Three cases that presented neurological deficits obtained significant improvement. Only one case evolved with pulmonary thromboembolic complications.

Conclusion The Schildhauer et al technique is an efficient technique for the fixation of complex sacral fractures with spinopelvic dissociation. The patients evolved with good functional results. Early weight-bearing has been shown to be safe with the use of this treatment.

\section{Introdução}

As fraturas do sacro correspondem a $1 \%$ de todas as fraturas da coluna vertebral, e, na maioria das vezes, estão associados a lesões do anel pélvico. Aproximadamente $60 \%$ das fraturas sacrais passam despercebidas no atendimento inicial do paciente. Elas geralmente são causadas por traumas de alta energia, como acidentes automobilísticos, quedas de altura, e tentativas de suicídio, ${ }^{1,2}$ estas também conhecidas como suicidal jumper's fractures ("fraturas do saltador suicida").

Inicialmente, as fraturas do sacro foram classificadas por Denis em lesões do tipo I, II e III. Em complemento à classificação de Denis, Isler classificou o tipo II em subtipos A, B e C, com relação ao traço de fratura através da faceta articular de L5-S1. Posteriormente, Roy-Camille descreveu o tipo III com traço transverso, conforme o mecanismo de trauma: flexão, extensão e cominutiva. Finalmente, as fraturas que envolvem ambos os lados do sacro podem ser classificadas conforme se assemelham a letras dos alfabetos latino e grego (U, T, H e $\lambda$ [lambda] $)^{1,3,4}$ ( - Figura 1). ${ }^{5}$ Outra forma de classificar as fraturas do sacro é por meio da classificação Arbeitsgemeinschaft für Osteosynthesefragen (AO, "Associação para o Estudo da Fixação Interna", em alemão) Spine. ${ }^{6}$
O tratamento varia de acordo com a estabilidade da fratura. Pode ser realizado tratamento conservador com repouso e retirada da carga axial sobre o sacro. 0 tratamento cirúrgico pode ser realizado de diferentes formas: parafusos e placas sacrais, e, nos casos de fraturas complexas do sacro com instabilidade vertical, é necessário realizar estabilização cirúrgica entre a coluna lombar e a pelve com sistemas que oferecem maior estabilidade. ${ }^{1,4}$

Na técnica de Schildhauer et $\mathrm{al}^{7}$ para inserção do parafuso de osso ilíaco, a asa do ilíaco é exposta parcialmente. Após isso, é identificado o ponto de entrada do parafuso $1 \mathrm{~cm}$ abaixo da espinha ilíaca posterossuperior. Neste ponto, realiza-se uma osteotomia de $1,5 \mathrm{~cm}$ para acomodar a cabeça do parafuso no ilíaco. Com um perfurador, ou procurador de pedículo, direciona-se o túnel em uma trajetória caudal de $45^{\circ}$ e lateral de $35^{\circ}$, apontando o perfurador ou o procurador de pedículo para a incisura glútea superior. Após a confirmação da trajetória com auxílio do fluoroscópio, o parafuso é inserido, sendo que, de acordo com a técnica utilizada, ele deve ter no mínimo $70 \mathrm{~mm}$ de comprimento 7,8 (-Figura 2).

Este trabalho tem por objetivo revisar a literatura médica existente e relatar a experiência de um serviço terciário de trauma no tratamento de uma série de casos de fratura sacral

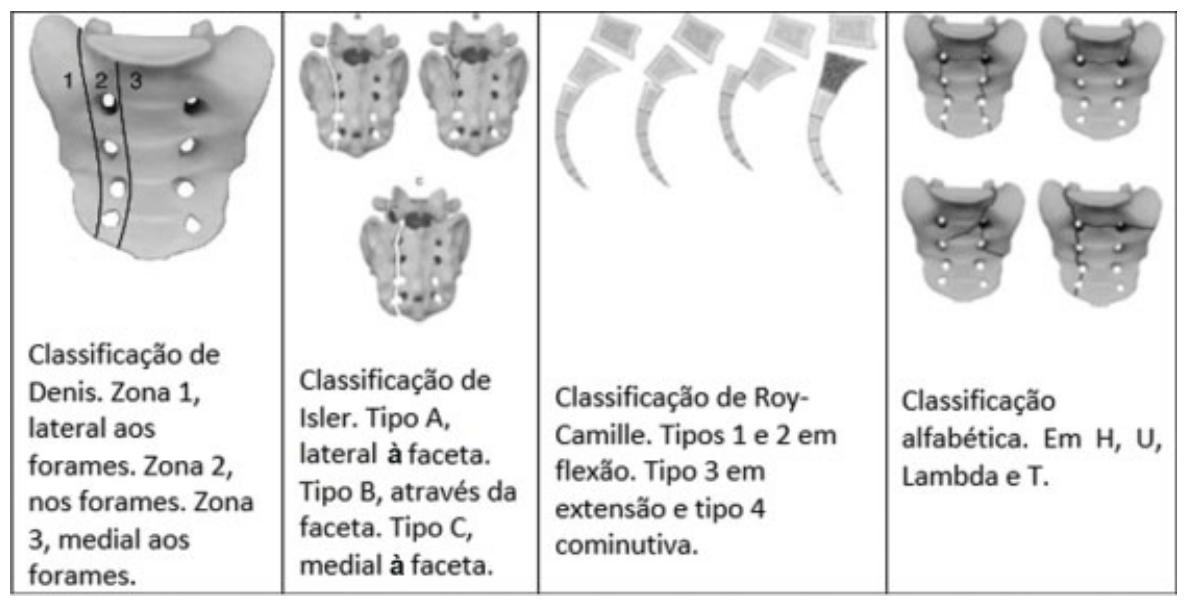

Fig. 1 Diferentes classificações de fratura sacral. Reproduzido de: Vaccaro et al. ${ }^{5}$ 


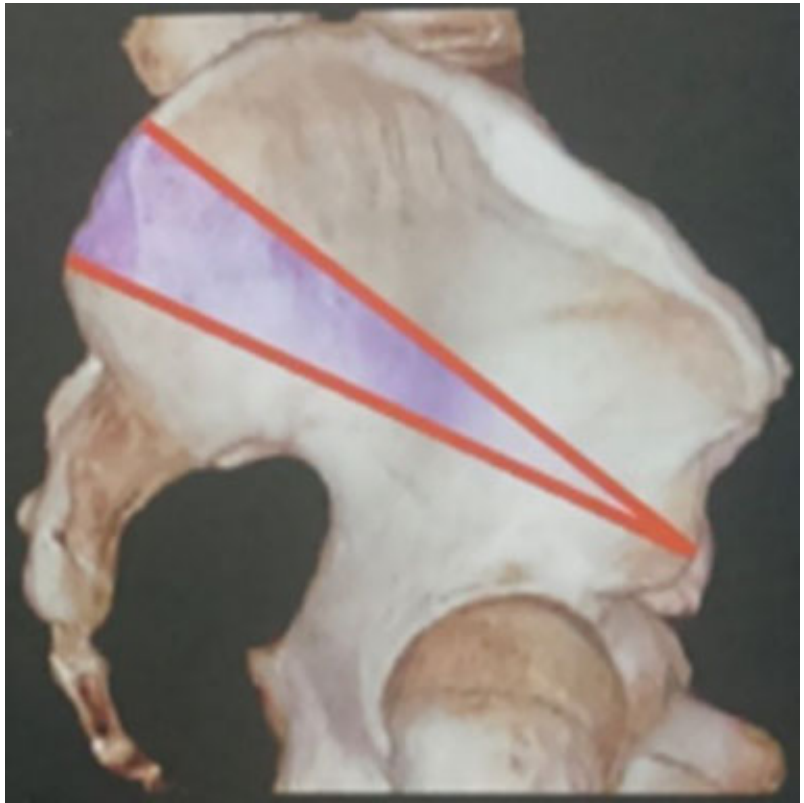

Fig. 2 Trajeto do parafuso de ilíaco pela técnica de Schildhauer et al. ${ }^{7}$ Reproduzido de: Schildhauer et al. ${ }^{7}$

com instabilidade vertical em que foi realizada a fixação espinopélvica, utilizando a técnica de Schildhauer et al $^{7}$ para a inserção dos parafusos do ilíaco.

\section{Método}

Este é um estudo retrospectivo, em que foi realizada uma análise da evolução clínica de uma série de casos, bem como de suas complicações, e os resultados desta análise foram comparados aos da literatura médica disponível atualmente.

O estudo foi aprovado pelo Comitê de Ética do hospital universitário sob o número 2.661.464.

Foram selecionados seis casos de fraturas complexas de sacro com instabilidade vertical, em que se optou pelo tratamento cirúrgico com fixação espinopélvica pela via posterior. Para a escolha da técnica e planejamento cirúrgico, foram realizadas radiografias nas incidências anteroposterior (AP), de perfil ( $\mathrm{P}$ ) do sacro, inlet e outlet da bacia, além de tomografias com reconstruções tridimensionais (-Figura 3). Por meio de uma abordagem mediana, foram expostos os níveis de L4 e L5 da coluna vertebral, o sacro e ambos os ilíacos para a instrumentação. Nos casos com compressão nervosa, foram realizadas laminectomia e descompressão posterior com remoção de fragmentos do canal e liberação foraminal. Nas fraturas com

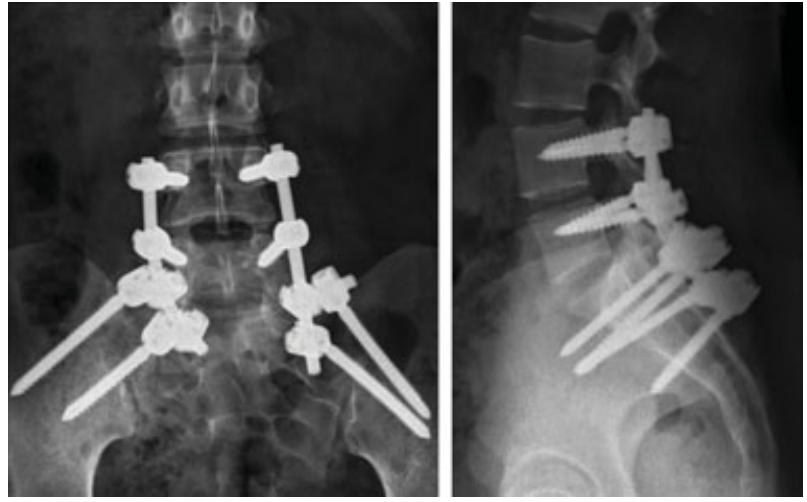

Fig. 4 Radiografias pós-operatórias nas incidências anteroposterior e de perfil demonstrando o sistema de fixação utilizado.

grande desvio ( $>1 \mathrm{~cm}$ ) foi realizada a redução indireta com o auxílio de pinças de redução, tração e pinos de Schanz como joysticks. Foram inseridos parafusos pediculares em L4, L5, S1 (quando o traço de fratura permitiu), e dois parafusos em cada ilíaco pela técnica de Schildhauer et $\mathrm{al}^{7}$ ( - Figura 4). ${ }^{7,8}$

Os critérios de inclusão foram: casos de fraturas do sacro, com instabilidade vertical e dissociação espinopélvica, com indicação de tratamento cirúrgico, em vítimas de trauma de alta energia, com ou sem lesões associadas, independente do sexo e do tipo da fratura, nos quais a opção cirúrgica foi a fixação espinopélvica pela técnica supracitada. Foram excluídos do estudo os casos de fraturas sacrais que não apresentavam instabilidade lombo pélvica, os que não apresentavam indicação cirúrgica, os tratadas com fixação diferente da fixação espinopélvica, e as fraturas patológicas causadas por mecanismos de baixa energia.

Após a seleção dos pacientes, foram analisados fatores epidemiológicos, como sexo e idade. Outros fatores analisados foram: presença ou ausência de lesão neurológica; lesões associadas; mecanismo do trauma; complicações clínicas relacionadas ao trauma; complicações relacionadas ao procedimento cirúrgico e ao tratamento; e a evolução clínica com segmento mínimo de 12 meses. O acompanhamento ambulatorial deu-se conforme os retornos regulares: após 15 dias da cirurgia, após 45 dias, após 90 dias, após 180 dias do procedimento, e retorno semestral após esse período, caso não houvesse particularidades do caso. A avaliação clínica foi baseada em dados registrados nos prontuários e nas pontuações no índice de incapacidade de Oswestry, ${ }^{9}$ versão 2.0 , e na escala visual analógica (EVA) da dor, que, como protocolo do serviço, são aplicados em todos os casos operados.
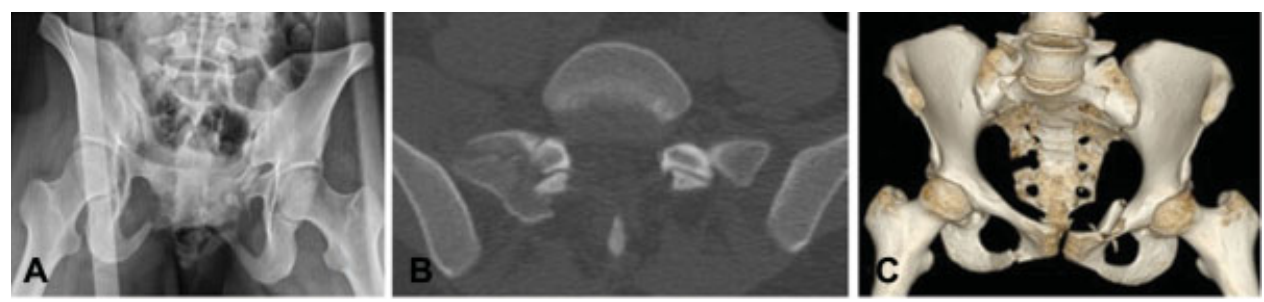

Fig. 3 Exames de imagem complementares. a) Radiografia na incidência outlet da bacia; b) tomografia axial computadorizada (TAC) com instabilidade espinopélvica e fratura do processo articular L5-S1; c) reconstrução tridimensional (3D) da TAC. 


\section{Análise de casos}

Entre os 6 casos analisados nesta série, 3 (50\%) foram em consequência de acidentes automobilísticos, e 3 (50\%) por tentativa de suicídio. A média de idade foi de 29 anos (variação: 20 a 48 anos), sendo 4 pacientes (66,6\%) do sexo feminino e $2(33,4 \%)$ do sexo masculino. A lesão associada mais comum foi a fratura de pelve, em $66,6 \%$ dos casos. Outras lesões encontradas foram: fratura de rádio distal, fratura de processos transversos da coluna lombar, trauma abdominal, e fratura de fêmur. Estas tiveram uma incidência menor ( 2 casos; 33,4\%) (-Tabela $\mathbf{1}$ ).

Apesar de todos os casos que deram entrada no prontosocorro terem sido classificados como Frankel $\mathrm{E},{ }^{10}$ dois destes apresentavam parestesia perineal, e um caso apresentava síndrome de far-out, ${ }^{11}$ nome dado à compressão da raiz de L5 entre o processo transverso de L5 e o fragmento fraturado do sacro, descrita inicialmente por Wiltse em 1984 , e observada mais comumente nas fraturas tipo II de Denis. ${ }^{3}$

Ao classificar as fraturas de sacro, verificou-se que metade dos casos foram classificados por Denis como de tipo 2, e metade, como de tipo 3. Dentre as de tipo 2 de Denis, 2 casos foram classificados como Isler tipo B. Utilizando a classificação descritiva, quando aplicável, foram encontradas duas fraturas em "U" e uma em "H". De acordo com a classificação da AO Spine, não foi identificado um padrão dominante.

Após a realização do procedimento de fixação, todos os pacientes foram mobilizados precocemente, permitindo-se que eles se sentassem no leito no primeiro dia de pósoperatório e praticassem mobilização ativa e passiva com exercícios de fisioterapia. Já no segundo dia de pós-operatório o paciente foi estimulado a se levantar com o auxílio de
Tabela 2 Resultados funcionais dos casos estudados

\begin{tabular}{|l|l|l|l|l|l|}
\hline EVA & Oswestry & Denis & Isler & Descritiva & AO \\
\hline 3 & $24 \%$ & Tipo 2 & Tipo B & Não se aplica & C2 \\
\hline 2 & $30 \%$ & Tipo 2 & Tipo C & Não se aplica & C2 \\
\hline 3 & $28 \%$ & Tipo 3 & Não se aplica & em U & C0 \\
\hline 4 & $50 \%$ & Tipo 3 & Não se aplica & em H & C3 \\
\hline 1 & $0 \%$ & Tipo 2 & Tipo B & Não se aplica & B2 \\
\hline 3 & $36 \%$ & Tipo 3 & Não se aplica & em U & C0 \\
\hline
\end{tabular}

Abreviaturas: AO, Arbeitsgemeinschaft für Osteosynthesefragen; EVA, escala visual analógica.

Nota: Fonte: Prontuário Eletrônico do Hospital do Trabalhador.

andadores de acordo com a limitação álgica e as limitações das lesões associadas.

Os casos foram acompanhados ambulatorialmente, com retornos pós-cirúrgicos em 15 dias, 45 dias, 3 meses, 6 meses, e 12 meses após o procedimento, e retorno semestral até a atualidade. $\mathrm{O}$ acompanhamento variou entre 12 e 24 meses, tendo início na data do procedimento cirúrgico.

\section{Resultados}

Os três pacientes com alteração neurológica que necessitaram de descompressão apresentaram recuperação completa no seguimento (dois casos com parestesia perineal e um com síndrome de far-out).

No índice de incapacidade de Oswestry, versão 2.0, 1 paciente foi classificado como tendo incapacidade mínima, 4 deles com incapacidade moderada, e 1 com incapacidade grave (-Tabela 2 ). 0 pior escore foi $50 \%$, de uma paciente que

Tabela 1 Características dos pacientes

\begin{tabular}{|c|c|c|c|c|}
\hline Sexo & Idade & Mecanismo & Lesões associadas & $\begin{array}{l}\text { Tempo de } \\
\text { acompanhamento }\end{array}$ \\
\hline Feminino & 20 & Colisão entre motocicleta e automóvel & Fratura bilateral dos ramos isquiopúbicos & 15 meses \\
\hline \multirow[t]{4}{*}{ Feminino } & 26 & Colisão entre motocicleta e automóvel & Fratura da pelve & \\
\hline & & & Fratura bilateral dos ramos isquiopúbicos & 12 meses \\
\hline & & & Disjunção de anel pélvico & \\
\hline & & & Hérnia diafragmática traumática & \\
\hline \multirow[t]{2}{*}{ Feminino } & 21 & Tentativa de suicídio (15 m) & Fratura cirúrgica em T12 & 24 meses \\
\hline & & & Lesão do complexo ligamentar posterior lombar & \\
\hline \multirow[t]{5}{*}{ Feminino } & 33 & Tentativa de suicídio (8 m) & Fratura bilateral dos ramos isquiopúbicos & 12 meses \\
\hline & & & Abertura de sínfise púbica & \\
\hline & & & Fratura subtrocantérica direita & \\
\hline & & & Fratura dos processos transversos T11 a L5 & \\
\hline & & & Fratural distal do rádio esquerdo & \\
\hline \multirow[t]{2}{*}{ Masculino } & 26 & Tentativa de suicídio (30 m) & Luxação subtalar exposta & 12 meses \\
\hline & & & Fratura do processo transverso L5 & \\
\hline \multirow[t]{3}{*}{ Masculino } & 48 & Colisão entre automóveis & Fratura dos ramos isquiopúbicos & 16 meses \\
\hline & & & Fratura do punho direito & \\
\hline & & & Fratura do cotovelo esquerdo & \\
\hline
\end{tabular}

Nota: Fonte: Prontuário Eletrônico do Hospital do Trabalhador. 
tentou suicídio e que apresentava fibromialgia e depressão grave, e era refratária ao tratamento psiquiátrico.

Como complicações, 1 paciente evoluiu com trombose venosa profunda no $21^{\circ}$ dia de pós-operatório, e 1 paciente, com deiscência de sutura, ambos com melhora após terapia adequada.

Durante o período de acompanhamento de 24 meses, foi necessária a retirada do material de síntese em 2 casos $(33,4 \%)$ devido a complicações já descritas na literatura: ${ }^{12}$ o primeiro, devido à soltura do material; o segundo, devido à proeminência e dor sobre o material de implante devido ao baixo índice de massa corporal.

\section{Discussão}

As fraturas complexas de sacro com instabilidade lombopélvica são graves, com múltiplas lesões associadas, e alto índice de morbidade e mortalidade. Mesmo quando tratadas de forma correta, devolvendo a estabilidade espinopélvica e evoluindo com a consolidação da fratura, os pacientes que sofreram este tipo de lesão podem cursar com sequelas da própria lesão ou do tratamento instituído. De acordo com a literatura, menos de $50 \%$ dos pacientes que sofreram fraturas complexas de sacro retornam às suas condições de trabalho e funcionalidades prévias. ${ }^{13}$

Apesar de serem consideradas raras, ${ }^{14,15}$ as fraturas causadas por tentativas de suicídio perfizeram $50 \%$ dos casos analisados neste estudo, mostrando-se uma parcela significativa das fraturas complexas de sacro.

Após a análise dos casos operados em nosso serviço, até o momento obtivemos resultados superiores aos descritos na literatura com um acompanhamento evolutivo dos pacientes. Foi observada uma boa evolução clínica, com poucos pacientes apresentando dor residual, e bons resultados cirúrgicos, com boa recuperação da função no curto e médio prazos. Cabe salientar que foram analisadas tanto artigos que utilizaram a mesma técnica cirúrgica (fixação iliolombar com parafusos de ilíaco pela técnica de Schildhauer et al $^{7}$ ), quanto aqueles que diferiram apenas na técnica do parafuso do ilíaco. Quanto às complicações cirúrgicas, tivemos um caso de soltura do material de síntese e um caso de infecção do sítio cirúrgico, ambos solucionados com desfecho satisfatório. A literatura nos mostra que essas complicações, tanto clínicas quanto cirúrgicas, são de grande incidência nos pacientes submetidos ao tratamento cirúrgico com fixação espinopélvica. ${ }^{15,16}$

Apesar da gravidade, risco de morte e morbidade envovlidos na fratura de sacro, observou-se boa evolução com a fixação espinopélvica nesta série de casos. Três casos foram em consequência de acidentes automobilísticos, e três, por tentativa de suicídio, o que corrobora os dados da literatura. ${ }^{1,2}$

A fixação espinopélvica é uma boa técnica de síntese para as fraturas de sacro com dissociação lombopélvica, pois permite mobilização imediata, bem como a sustentação de carga no pós-operatório. ${ }^{17}$ Nesta série, todos os casos evoluíram com consolidação completa, e não observamos perda de redução da fratura em consequência da sustentação de carga e da mobilidade imediata.
Tendo em vista as diferentes técnicas para fixação, optou-se pela técnica de fixação espinopélvica com parafusos pediculares associados a duas hastes de titânio, que, comparada às técnicas citadas na literatura, como a osteossíntese rígida com placas e parafusos e a osteossíntese com banda de tensão posterior, possibilita a liberação de sustentação de carga e movimentos precoces, apresenta vantagem sobre os parafusos iliossacrais percutâneos, provendo maior estabilidade vertical ao sistema de fixação, ${ }^{18}$ e até $25 \%$ a mais de resistência quando comparada à fixação associada com apenas uma haste. ${ }^{19}$ Levando em consideração as diferentes técnicas de inserção dos parafusos no ilíaco, a literatura apresenta a técnica de Schildhauer et al $^{7}$ como superior em relação às técnicas de Berry e de Miller, pois a triangulação convergente dos parafusos na primeira fornece uma maior resistência tensional e rotacional.

De acordo com o estudo de Guimarães et al, ${ }^{20}$ uma opção seria o tratamento com fixador externo supra-acetabular associado a parafusos sacroilíacos percutâneos; entretanto, os casos desse estudo eram especificamente fraturas classificadas como Tile tipo $C$, não havendo inclusão de outros tipos de fraturas complexas de sacro na análise.

Segundo a EVA de dor, quatro foi a maior pontuação, sendo que a dor melhorou com o uso de analgésico simples.

Analisando a evolução funcional por do pindice de incapacidade de Oswestry, versão 2.0 , o pior resultado foi $50 \%$, verificado em apenas 1 (16,6\%) dos casos, o que denota uma incapacidade funcional grave; os demais casos foram classificados como disfunções mínima e moderada.

Quanto ao caso em nossa série que apresentou síndrome de far-out, foi realizada a descompressão da raiz L5 por via posterior além da fixação espinopélvica, levando a um bom resultado clínico, com alívio dos sintomas de compressão da raiz. Este fato corrobora as técnicas comumente usadas no tratamento dessa patologia, quando encontrada. ${ }^{17}$

Entre as complicações comuns associadas à fixação, há a dor sobre os implantes devido à saliência do parafuso e à soltura do material de síntese em longo prazo. Três pacientes apresentaram dor sobre o material de síntese, sendo que um deles apresentava consolidação e sinais de soltura nos parafusos do ilíaco bilateral, e, em outro caso, os parafusos tornaram-se salientes após perda ponderal do paciente, sendo necessária a retirada dos parafusos. Os demais não apresentaram tais complicações até o momento. 2,16,20

É provável que retirar eletivamente o material de síntese após a consolidação da fratura diminua a dor residual no local da cirurgia; entretanto, devemos lembrar que. por se tratar de uma lesão grave e de difícil tratamento, a dor referida pode não ser proveniente somente do material, podendo ser sequela da própria lesão.

Nos pacientes em que o material foi retirado, houve uma melhora significativa da dor residual na região lombar baixa, tendo em vista que a localização superficial do material de síntese causava a sensação dolorosa na posição sentada.

Visto que na literatura se afirma que a maioria das complicações relacionadas ao material de síntese ocorrem dois anos após o procedimento, ainda podemos esperar que haja soltura do material de síntese tardiamente, principalmente após a consolidação da fratura sacral e da artrodese espinopélvica. 


\section{Conclusão}

Mais de $80 \%$ dos casos acompanhados demonstraram resultado satisfatório em médio prazo com a técnica de fixação utilizada. Isso sugere que a técnica de Schildhauer et al $^{7}$ para fixação espinopélvica é segura e com boa resistência para liberação de carga no pós-operatório imediato.

Entretanto, por se tratar de um estudo retrospectivo que avaliou um número limitado(6) de pacientes tratados somente em um serviço, novos estudos prospectivos e com uma amostra maior devem ser conduzidos para se chegar a conclusões melhores sobre a eficácia e segurança da técnica empregada.

Conflito de Interesses

Os autores declaram não haver conflitos de interesse.

\section{Referências}

1 Keith DW. Fractures, dislocations, and fracture-dislocations of the spine. In: Canale ST, Beaty JH, editors. Campbell's operative orthopaedics. 12th ed. Canada: Elsevier; 2013:1559-1627

2 Vilela MD, Jermani C, Braga BP. Lumbopelvic fixation and sacral decompression for a U-shaped sacral fracture: case report. Arq Neuropsiquiatr 2007;65(3B):865-868

3 Bellabarba C, Schildhauer TA, Chapman JR. Sacral frantures. In: Herkowitz HN, Garfin SR, Eismont FJ, Bell GR, Balderston RA, editors. Rothman-Simeone The spine. 6th ed. Philadelphia: Saunders/Elsevier; 2013:1405-1421

4 Schroeder GD, Savage JW, Patel AA, Stover MD. Spinopelvic fixation in complex sacral fractures. JBJS Rev 2015;3(03):e4

5 Vaccaro AR, Kim DH, Brodke DS, et al. Diagnosis and management of sacral spine fractures. Instr Course Lect 2004;53(01):375-385

6 Sacral Trauma [acesso em 2018 abril 18]. Disponível em: https:// www2.aofoundation.org/wps/portal/surgerymobile?showPage= diagnosis\&bone=Spine\&segment=TraumaSacrum

7 Schildhauer TA, Ledoux WR, Chapman JR, Henley MB, Tencer AF, Routt MLJr. Triangular osteosynthesis and iliosacral screw fixation for unstable sacral fractures: a cadaveric and biomechanical evaluation under cyclic loads. J Orthop Trauma 2003;17(01):22-31

8 Rhee WT, You SH, Jang YG, Lee SY. Lumbo-sacro-pelvic fixation using iliac screws for the complex lumbo-sacral fractures. J Korean Neurosurg Soc 2007;42(06):495-498
9 Fairbank JC, Pynsent PB. The Oswestry Disability Index. Spine 2000;25(22):2940-2952, discussion 2952

10 Maynard FM Jr, Bracken MB, Creasey G, et al; American Spinal Injury Association. International Standards for Neurological and Functional Classification of Spinal Cord Injury. Spinal Cord 1997; 35(05):266-274

11 Ise S, Abe K, Orita S, et al. Surgical treatment for far-out syndrome associated with abnormal fusion of the L5 vertebral corpus and L4 hemivertebra: a case report. BMC Res Notes 2016;9:329

12 Rocha VM, Guimarães JA, Olivaes Filho AP, et al. Sacral fracture treatment with a variation of the lumbopelvic fixation technique. Coluna/Columna 2018;17(01):69-73

13 Bucholz RW, Heckman JD, Court-Brown CM, Torneta P 3rd. Rockwood and Green's Fractures in adults. 7th ed. Philadelphia: Lippincott Williams \& Wilkins; 2009

14 Lee SW, Ko SB, Shin DY. Sacral Fracture Dislocation of Suicidal Jumper (Suicidal Jumper's Fracture) - 4 Cases Report -. J Korean Soc Spine Surg 2008;15(04):281-285

15 Roy-Camille R, Saillant G, Gagna G, Mazel C. Transverse fracture of the upper sacrum. Suicidal jumper's fracture. Spine 1985;10(09): 838-845

16 König MA, Jehan S, Boszczyk AA, Boszczyk BM. Surgical management of U-shaped sacral fractures: a systematic review of current treatment strategies. Eur Spine J 2012;21(05):829-836

17 Schildhauer TA, Bellabarba C, Nork SE, Barei DP, Routt ML Jr, Chapman JR. Decompression and lumbopelvic fixation for sacral fracture-dislocations with spino-pelvic dissociation. J Orthop Trauma 2006;20(07):447-457

18 Kaye ID, Yoon RS, Stickney W, Snavely J, Vaccaro AR, Liporace FA. Treatment of Spinopelvic Dissociation: A Critical Analysis Review. JBJS Rev 2018;6(01):e7

19 Ueno FH, Pisani MJ, Machado AN, Rodrigues FL, Fujiki EN, Rodrigues LMR. Estudo biomecânico da fixação da fratura sacroilíaca com barras de titânio e parafusos pediculares. Acta Ortop Bras 2015;23(03):154-157

20 Guimarães RP, Ribeiro AG, Ulson O, Ávila RB, Ono NK, Polesello GC. Tratamento das lesões instáveis do anel pélvico com fixador supra-acetabular e parafusos sacroilíacos: Resultados preliminaries em 20 pacientes. Rev Bras Ortop 2016;51(02): 132-137

21 Sagi HC. Technical aspects and recommended treatment algorithms in triangular osteosynthesis and spinopelvic fixation for vertical shear transforaminal sacral fractures. J Orthop Trauma 2009;23(05):354-360 